Archives

12 | 1994

Polysémie de la santé. Institutions et pratiques

sociales en France et au Québec 1750-1980

\title{
Le service de santé de Montréal de l'établissement au démantèlement (1865-1975)
}

Benoît Gaumer, Georges Desrosiers, Othmar Keel et Céline Déziel

\author{
(2) OpenEdition \\ Journals \\ Édition électronique \\ URL : http://journals.openedition.org/ccrh/2748 \\ DOI : $10.4000 /$ ccrh. 2748 \\ ISSN : 1760-7906 \\ Éditeur \\ Centre de recherches historiques - EHESS \\ Édition imprimée \\ Date de publication : 12 avril 1994 \\ ISSN : 0990-9141 \\ Référence électronique \\ Benoît Gaumer, Georges Desrosiers, Othmar Keel et Céline Déziel, «Le service de santé de Montréal \\ de l'établissement au démantèlement (1865-1975)», Les Cahiers du Centre de Recherches Historiques \\ [En ligne], 12 | 1994, mis en ligne le 27 février 2009, consulté le 04 mai 2019. URL : http:// \\ journals.openedition.org/ccrh/2748; DOI : 10.4000/ccrh.2748
}

Ce document a été généré automatiquement le 4 mai 2019.

Article L.111-1 du Code de la propriété intellectuelle. 


\title{
Le service de santé de Montréal de l'établissement au démantèlement (1865-1975)
}

\author{
Benoît Gaumer, Georges Desrosiers, Othmar Keel et Céline Déziel
}

1 Au cours de la décennie 1970, le Québec entreprend une vaste réforme de son système de santé qui aboutit au démantèlement des anciennes structures de santé publique mises en place à partir de 1926 comme les unités sanitaires de comté ou de la fin du XIX siècle comme le service de santé de la ville de Montréal. Cette étude, en cours et partie d'une enquête plus vaste sur les conditions d'institutionnalisation des pratiques de santé publique au Québec du milieu du XIX ${ }^{\mathrm{e}}$ s. à nos jours, analyse les raisons de la disparition de ce service de santé de la ville de Montréal en reconstituant la trame de son existence. L'histoire de l'organisation est replacée dans le contexte socioculturel et politique de Montréal et des époques successives.

2 La mise en parallèle des tendances séculaires de la mortalité entre 1871 et 1971 permet de porter un jugement sur l'impact des programmes de santé publique sur l'état de santé de la population de la ville. Il ressort qu'en dépit d'une comparaison défavorable avec certaines autres villes nord américaines, le Service de santé de Montréal a su consolider son organisation et étendre ses activités, contribuant à l'amélioration progressive de l'état de santé de la population. Le démantèlement de ce service en 1975 constitue donc un vrai paradoxe, d'autant qu'il s'est fait au nom d'une médecine globale mettant l'accent sur la prévention, qui était justement la visée première du Service de santé. Cet article tente d'élucider ce paradoxe.

\section{Introduction}

Comme la ville de New York qui institue de manière durable son premier bureau de santé en 1866, la ville de Montréal existe déjà depuis plus de deux siècles et demi lorsque son conseil municipal prend les moyens de mettre sur pied en 1865 une organisation 
semblable en lui assurant une continuité1. A plusieurs reprises antérieurement, devant la menace des grandes épidémies notamment de choléra, venant d'Europe, la ville avait créé des bureaux de santé éphémères qui ne duraient que pendant ces épidémies ou leur menace et qui disparaissaient aussitôt après. En outre, ces bureaux ou comités étaient mis en place presque toujours trop tard pour être efficaces et manquaient de moyens ou de détermination pour agir².

De sa fondation à 1865 , la ville est passée d'un petit poste de commerce de fourrures à une agglomération d'une centaine de milliers d'habitants. De 1867 (création de la Confédération canadienne) au début des années 1970, période qui voit la naissance, le développement, la maturité et la disparition du Service de santé de Montréal), la ville passe par plusieurs phases qui ont été bien analysées par les historiens ${ }^{3}$. De 1867 à 1896, la ville constitue son tissu industriel, et se dote des principales infrastructures qui orienteront pour longtemps son avenir. De 1896 à 1914, Montréal est à son apogée de métropole du Canada. Cette croissance rapide qui attire Québécois des campagnes environnantes et immigrants européens va se traduire cependant par des inégalités sociales et ethniques très marquées. Entre 1914 et 1945, les crises de croissance vont se multiplier, celle des années 1930 et des deux grandes guerres étant les plus marquantes. Comme partout ailleurs, au Canada et dans le monde industrialisé, l'après 1945 entraîne de profonds changements. L'agglomération atteint sa taille de grand centre urbain contemporain et sa population jouit d'un niveau de vie sans précédent.

Ces grandes périodes de l'histoire de Montréal nous serviront de trame pour analyser l'évolution de ce qui s'est appelé successivement Comité ou Bureau de santé, Commission d'hygiène, Bureau municipal d'hygiène et de statistiques, et finalement Service de santé à partir de 1918. Cette étude vise d'abord à saisir la spécificité et l'originalité de cette structure montréalaise d'intervention en santé publique qui a fonctionné pendant plus de cent ans et dont les racines s'enfoncent dans les courants plus anciens de la "police médicale » ou du « sanitary movement ».

6 Le Service de santé de Montréal (ainsi que l'état de santé de la population) ont été étudiés en partie, surtout pour la période des commencements ${ }^{4}$ et pour celle allant de 1880 à 1914 5. De plus Terry Copp a examiné dans les grandes lignes l'état de santé des montréalais et les activités sanitaires (en partie) pour la période $1897-1929^{6}$ mais ce dernier ne s'attarde pas aux dispositifs administratifs de santé publique, bien qu'il soit amené à parler du Service de santé. C'est donc une première esquisse des différentes étapes de toute l'évolution du Service de santé de Montréal que nous nous proposons de présenter dans cette communication. Il s'agit des résultats préliminaires d'une recherche entreprise depuis plus d'un an et qui va être poursuivie systématiquement pour chaque grande période. Des monographies approfondies ont déjà été consacrées à des services de santé publique de villes nord-américaines qui présentent un certain nombre de ressemblances avec Montréal comme New York ${ }^{7}$, Toronto ${ }^{8}$ ou Milwaukee ${ }^{9}$. Elles nous serviront de points de comparaison pour mieux repérer l'ancrage du service de santé dans son environnement urbain. Par ailleurs, cela nous permettra de dégager les spécificités de la structure montréalaise.

7 L'exposé des résultats préliminaires de cette recherche sera essentiellement chronologique: il retracera les grandes étapes de la vie de l'organisation. Une place importante est accordée à un essai d'analyse de l'évolution de l'état de santé de la population montréalaise. La confrontation d'indices démographiques et d'indicateurs de santé de la population avec une description minutieuse des interventions de santé 
publique devrait apporter un éclairage supplémentaire à la question controversée de l'impact réel des programmes et politiques socio-sanitaires sur les états de santé des populations ${ }^{10}$. Par ailleurs, nous nous proposons d'éclairer, dans cette étude une question majeure et qui ne laisse pas de surprendre : comment et pour quelles raisons en est-on venu à démanteler, dans les années 1970, un service bien organisé, que l'on avait construit et développé au prix de tant d'efforts, dont on avait si souvent déploré autrefois, d'abord l'inexistence, puis les insuffisances et qui était maintenant depuis longtemps largement reconnu non seulement comme utile, mais encore comme indispensable pour les dirigeants, les élites et la population en général? Mais il faut commencer par délimiter le champ d'action du Service de santé de Montréal, avant d'analyser cette action même, le fonctionnement de ses agents ainsi que l'évolution de sa structure et les aléas de son histoire.

\section{Cadre territorial et population de 1861 à 1971}

L'Ile de Montréal constitue un ensemble naturel sur le plan épidémiologique qui ne coïncide pas avec les découpages administratifs successifs de la ville, ce qui sera souvent dénoncé comme un obstacle important par les responsables de la surveillance sanitaire. Le territoire de départ de la ville est fixé par un décret qui date de $1792^{11}$. Les recensements successifs permettent de suivre l'évolution de cette population de 1861 à 1971. Le tableau I résume cette évolution en distinguant la ville elle-même qui correspond à l'aire de responsabilité du service de santé, objet de cette étude, et l'île qui bénéficie des autres services de santé municipaux qui s'en partagent le territoire. La carte 1 représente le territoire montréalais à la fin du xix ${ }^{e}$ siècle. $C^{\prime}$ est d'abord dans l'espace de base fixé par les limites de 1792 que se développe la population de la ville. A partir de 1883 celle-ci va annexer progressivement une série de municipalités de banlieue, agrandissant d'autant sa surface et bénéficiant de leur forte croissance démographique. Les dernières décennies du XIX ${ }^{e}$ siècle sont marquées par une croissance de la population montréalaise plus rapide que celle de l'ensemble du Québec. Alors qu'en 1871 la ville ne rassemblait que $9 \%$ de la population québécoise, à la fin du siècle, un cinquième de la population québécoise vit à Montréal. A cette époque l'expansion se fait principalement à partir d'une émigration interne de ruraux canadiens-français, provenant souvent des mêmes comtés surpeuplés que ceux dont les ressortissants vont grossir la main-d'œuvre des filatures de la NouvelleAngleterre. Cela va se traduire par un renforcement du groupe ethnique francophone dont la représentation passe de $53 \%$ en 1871 à $60.9 \%$ en 1901, se partageant alors avec le groupe anglophone $98 \%$ des effectifs de la population totale de la ville.

9 A partir du tournant du $\mathrm{xx}^{\mathrm{e}}$ siècle, l'immigration externe va reprendre avec une diversité accrue des pays d'origine. Les groupes qui ne sont pas d'origine francophone ou anglophone représentent $10 \%$ de la population montréalaise en 1911, les juifs constituant la moitié de ces effectifs. A nouveau la ville va se développer par annexions de municipalités avoisinantes en entier ou partiellement comme l'indique la carte 2. Cette diversification ethnique s'accompagne d'un éparpillement des zones de peuplement homogène plutôt que d'un réel cloisonnement géographique des communautés linguistiques ou culturelles.

Durant toute la période couverte par cette étude on assiste à un réaménagement permanent et parfois en profondeur de tout l'espace urbain qui va fixer les traits du Montréal d'aujourd'hui. Dans la dernière partie du xix ${ }^{e}$ siècle, le centre de Montréal, qui 
correspond au Vieux-Montréal d'aujourd'hui, devient un centre d'affaires et d'administration. Le district de Montréal Ouest, fief traditionnel des Montréalais anglophones est occupé de plus en plus par une minorité significative de Canadiens français. De plus, c'est dans ce district que se concentrent deux communautés plutôt pauvres, les Irlandais dans Sainte-Anne et les Juifs originaires d'Europe de l'Est dans Saint-Laurent. C'est le district de Montréal Est, fief principal des Montréalais francophones, qui va croître le plus rapidement, représentant à la fin du XIX ${ }^{\mathrm{e}}$ siècle $50 \%$ du peuplement de la ville. L'expansion de la banlieue qui débute dès les années 1870, puis l'immigration externe, qui reprend après le début $\mathrm{du} \mathrm{xx}^{\mathrm{e}}$ siècle, vont encore complexifier la mosaïque ethnique de peuplement qu'il est nécessaire de suivre pour tenter de saisir l'accessibilité des services du bureau municipal de santé et de ses différentes ressources à l'ensemble de la population du territoire.

11 Les recensements décennaux comme les estimations annuelles ne permettent pas de prendre en compte ce que les surintendants ou responsables successifs de la division de la démographie appellent la population flottante par rapport à la population de jure, c'est-àdire domiciliée à Montréal. Cette distinction revient régulièrement sous la plume des responsables du Service de santé qui cherchent à calculer de manière précise cette fraction de la population représentée de facto par les personnes habitant dans la ville depuis moins d'un an. Tous les indices démographiques et indicateurs sanitaires seront établis en utilisant la population de jure comme dénominateur. Ce sont ces limites changeantes de la population desservie et l'interpénétration de la ville et de sa banlieue qui expliquent les plaidoyers fréquents en faveur d'une autorité centrale pour le Service d'hygiène de l'île de Montréal c'est-à-dire d'une seule structure sanitaire.

\section{L'état sanitaire de Montréal vers 1860}

Les historiens, comme déjà les Montréalais de l'époque, ont tous souligné le caractère déplorable des conditions sanitaires de la vie à Montréal au XIX ${ }^{\mathrm{e}}$ siècle, particulièrement dans la seconde moitié du siècle, marquée par une croissance rapide de la population et les effets néfastes d'une urbanisation sauvage.

13 Sans doute, les préoccupations pour la santé publique n'étaient pas étrangères aux décennies antérieures. Le choléra de 1832 et 1834, le typhus de 1847, par exemple, avaient massivement sévi et provoqué des mobilisations. Ainsi chaque grande épidémie suscite-telle d'éphémères bureaux ou comités, mis en place presque toujours trop tard pour avoir quelque efficacité que ce soit et manquant ou des moyens ou de la détermination pour $\operatorname{agir}^{12}$.

14 A partir de 1850 , de nombreuses sources médicales font état d'une détérioration des conditions de vie et de santé des Montréalais. On s'inquiète, par exemple, de ce que Montréal, qui pourtant jouirait d'une situation topographique privilégiée sous le rapport sanitaire, présente une mortalité double de celle de Londres, pourtant beaucoup plus populeuse et que la nature aurait moins avantagée. Dès 1859, dans une des premières tentatives pour chiffrer des indicateurs de santé publique à Montréal, on croit pouvoir conclure que la mortalité, déjà en 1857 deux fois plus élevée que celle de Toronto (34 \%o au lieu de $15 \%$ ), s'accentue jusqu'à atteindre $38 \%$ en $1859^{13}$. Sur la base d'une analyse des registres des inhumations, le même auteur, huit ans plus tard, (critiquant les résultats du recensement de 1861 qui donne une mortalité montréalaise de 22,5\%o), conclut à une 
alarmante détérioration, les six années écoulées de 1860 à 1865 exhibant des taux atteints seulement dans les pires villes anglaises durant les périodes « of special pestilence». Ses taux de $68 \%$ en 1860 et de $86 \%$ en 1865 sont sûrement exagérés, mais témoignent des inquiétudes de l'époque.

A lire les témoignages des contemporains, d'ailleurs en tous points vérifiables dans les archives municipales, le Montréal de l'époque, sauf quelques coins privilégiés, n'a rien d'idyllique. Peu de rues sont pavées ou macadamisées, les pluies les transforment en bourbiers. Des cadavres d'animaux y pourrissent par centaines; les ordures s'empilent dans les rues, les ruelles et les cours, fournissant une nourriture à bon marché pour les porcs que plusieurs s'obstinent à élever en ville en dépit des règlements. Ces ordures, ramassées très irrégulièrement - presque jamais l'hiver - forment au dégel des congères malodorantes. Les trottoirs, là où ils existent, sont souvent de bois ; ils ont tendance à pourrir et à s'imbiber de fange. Le réseau des égouts demeure fort incomplet, bâti par à coups, sans plan d'ensemble; ses raccords sont souvent défectueux; ses pentes mal calculées, son diamètre par endroit insuffisant, en font une source de pollution et d'infestation des quartiers bas de l'est. Le water-closet n'est pas encore une commodité courante, et des dépôts de matières fécales s'enfouissent dans les cours et autour des maisons. En somme, et surtout à une époque où l'étiologie miasmatique restait dominante, ces spectacles quotidiens auraient dû suffire pour inciter à l'intervention.

La mise en place d'un dispositif de recueil et analyse des statistiques mortuaires sous la responsabilité des officiers de santé de la cité de Montréal va constituer le noyau central autour duquel vont se structurer progressivement les activités du bureau ou Service de santé. Durant la décennie 1870, le taux brut de mortalité générale oscille entre 30 et 37 pour 1000 habitants amorçant cependant une baisse à partir de 1878, ce qui coïncide avec une première réorganisation du bureau de santé de la ville (voir plus loin section 4). Les études concernant la mortalité infantile à Montréal au milieu du XIXe siècle indiquent des taux autour de 250 décès pour 1000 enfants nés vivants confirmant cet état dramatique de la situation sanitaire de la cité lorsque le bureau d'hygiène et de statistique apparaitt ${ }^{14}$.

\section{Les commencements d'une administration montréalaise de la santé publique}

Jusqu'à 1840, semble-t-il, les grandes fonctions d'hygiène publique ou de police médicale relèvent directement des autorités municipales. Déjà, à la suite de la loi de 1832 pour l'établissement de bureaux sanitaires, un bureau sanitaire est formé la même année mais il est dissout l'année suivante. A partir de cette époque cependant des commissions échevinales apparaissent comme la Commission de police et de santé qui recommande elle-même dès 1847 l'institution d'un premier bureau de santé dont l'existence sera encore une fois éphémère.

La création de jure d'un bureau de santé permanent date de 1865, provoqué là encore, semble-t-il, par la menace de choléra qui sévit en Europe. Cette fois-ci les autorités prennent le devant et surtout les deux officiers de santé recrutés vont demeurer en fonction après l'alerte épidémique ${ }^{15}$. La Montreal Sanitary Association, mise sur pied en 1866, va plaider en faveur du maintien de fonctionnaires publics pour pallier le caractère changeant des comités de santé. Dans cette association, présidée par William Workman qui allait devenir maire de Montréal en 1868, on retrouve le docteur Larocque, un des 
deux officiers de santé nommé en 1866 et qui était devenu, de ce fait, le premier "Médecin de la cité de Montréal » à plein temps. L'association était patronnée par un comité comprenant Monseigneur Bourget, l'évêque anglican Fulford, le principal de McGill William Dawson et une brochette d'hommes d'affaires, hommes politiques et notables dont environ $20 \%$ de francophones ${ }^{16}$.

La nomination des deux officiers de santé qui ne font pas partie du comité de santé ne règle pas pour autant les difficultés de ce dernier à coordonner les actions d'hygiène publique et de police médicale qui sont dispersées entre les différents comités, en particulier celui des marchés, des chemins et de l'aqueduc.

En 1875, dans un contexte de variole endémique, une nouvelle épidémie très sévère frappe la ville, ce qui, en raison de la situation sanitaire, déjà reconnue comme très précaire par les élites et la profession médicale, favorise l'accession à la mairie d'un notable déjà connu pour son engagement en faveur de la santé publique et ses compétences, le fameux chirurgien William Hingston.

En 1874, la ville avait obtenu une charte complètement refondue qui lui permettait de règlementer de nombreux domaines de la santé au travers de son Bureau de santé. A la faveur de l'émoi causé par la nouvelle épidémie de variole, le Conseil municipal parvenait à imposer, au début de 1875, un amendement à la nouvelle charte, renforçant les pouvoirs des officiers de santé et les autorisant «à faire des visites domiciliaires, à détruire les hardes, vêtements et tout article infecté de la variole ou toute autre maladie contagieuse, à effectuer l'isolement des patients atteint de ces maladies quand les dits officiers le jugeront nécessaire au bien-être des dits patients ou du public en général, à faire effectuer l'inhumation à bref délais de toute personne décédée par suite des dites maladies, enfin de prendre telles mesures que le conseil de la dite cité jugera nécessaires, pour régler, contrôler, prévenir ou arrêter les progrès de la variole ou autre maladie contagieuse, épidémique ou pestilentielle dans la dite cité, nonobstant toute loi actuellement en force à ce contraire ${ }^{17}$ ».

S'appuyant sur ce dispositif juridique, Hingston allait, au cours de ses deux mandats, procéder à une réorganisation en profondeur de l'administration sanitaire et de sa réglementation. En 1870, sous William Workman, le Conseil municipal avait voté le règlement 37 établissant un bureau de santé, présidé par le maire et formé par des membres des Comités de santé et de police, responsable de la mise en œuvre des mesures de police sanitaire. Par les règlements 104 et 105 de 1876, l'administration Hingston maintenait le Bureau ainsi formé en 1870. Mais, comme il ne s'était jamais réuni plus de trois ou quatre fois l'an, on lui adjoignait un sous-comité plus restreint, où le maire et les officiers de santé pouvaient eux-mêmes jouer un rôle majeur, auquel était dévolu la charge réelle de la police sanitaire. Ce comité siégeait chaque semaine. Le règlement 37 de 1870 autorisait le Bureau de santé à s'adjoindre en cas d'urgence épidémique des membres de l'extérieur pour la durée de la crise. Avec le règlement 105, il devenait permanent que des membres de l'extérieur du Conseil fussent nommés au Bureau, ce qui eut pour conséquence de renforcer la présence médicale dans l'administration sanitaire. $\mathrm{Au}$ lieu qu'en 1865 et en 1870 l'expertise médicale paraissait ne s'imposer qu'en situation épidémique, les mesures de 1876 posaient sa pertinence dans la conception et la mise en oeuvre régulière de la politique sanitaire.

Avec Hingston, la réglementation sanitaire avait donc obtenu les moyens, parfois draconiens, de son application réelle; les officiers de santé et inspecteurs municipaux, dans certaines limites, allaient pouvoir procéder de façon coercitive, et ce bien que 
certains aient dénoncé déjà les pouvoirs généraux consentis dans la charte de 1874 comme « une atteinte portée à la liberté individuelle».

L'offensive du maire trouvait des appuis importants dans les élites et certains groupes de pression, surtout anglophones, comme la Citizen Public Health Association créée en 1875. Convoquée et présidée par Hingston lui-même, la nouvelle association rassemblait des médecins et des citoyens intéressés à la chose publique, et parmi eux des notables comme le principal de McGill, William Dawson, William Osler, et plusieurs membres du clergé, dont l'évêque anglican de Montréal. Cette association paraît renouer avec la Montreal Sanitary Association. De fait, en plus de ses nombreuses interventions, la Citizen Public Health Association a diffusé et popularisé systématiquement pendant deux ans (1875-1877) les idées et les directives de la nouvelle politique sanitaire municipale, à travers une revue spécialisée qu'elle avait lancée, le Public Health Magazine.

La grande mobilisation coïncidant avec l'arrivée de Hingston à la mairie, en redonnant vigueur au mouvement provoqué d'abord par le maire Workman, consolidait considérablement l'administration sanitaire et augmentait notablement les pouvoirs de ses agents en même temps qu'elle donnait à la profession médicale un poids qu'elle n'avait jamais eu dans la gestion de la citén ${ }^{18}$.

La structure et la politique sanitaires mises en place par Hingston et les forces qui l'appuyaient (en particulier l'officier de santé de la ville, Larocque), vont servir de base, malgré certains atermoiements et certains reculs sous le mandat de son successeur, le maire Beaudry, aux développements ultérieurs du service de santé et de l'action sanitaire municipale. Malgré le revirement du nouveau maire Beaudry, à partir de 1877, qui considérait que l'on avait accordé une place trop importante aux questions sanitaires dans les affaires municipales, le maintien en fonction de Larocque, au poste stratégique d'officier de santé, tout comme les activités sanitaires des diverses instances administratives (comité des chemins, des marchés, etc.) ont permis d'assurer une certaine continuité jusqu'en 1885, où sous le nouveau maire Beaugrand, les questions sanitaires ont été reconnues à nouveau expressément comme une priorité absolue de la politique municipale. A noter aussi que sous le mandat du maire S. Rivard (1879-1881), ce dernier a appuyé Larocque et valorisé le travail du Bureau de santé et de ses officiers pour le bon état sanitaire de la ville en 1881 et l'efficacité de la vaccination.

Sous son deuxième mandat (1881-1884), Beaudry semble moins militer contre le service de santé. Il faut tenir compte du contexte politique général à Montréal et au Québec pour comprendre l'attitude de Beaudry en 1877 lorsqu'il avait succédé à Hingston. Beaudry avait des comptes à régler avec ce dernier, lequel l'avait battu à une majorité écrasante à l'élection de 1875. Il y avait d'autre part un clivage politique et ethnique entre francophones et anglophones. Beaudry était un ancien patriote et un membre du parti conservateur qui représentait l'élite nationaliste francophone. La campagne de Hingston pour la santé publique avait été menée surtout par une élite anglophone de 1875 à 1877 tout comme l'avait été celle de Workman quelques années plus tôt. Il est donc assez compréhensible que Beaudry, pour des raisons partisanes, se soit abstenu de valoriser les réalisations de son prédécesseur et d'en vanter les mérites; il s'est même employé à les dénigrer et à les contrecarrer, sans parvenir, cependant, à remettre en cause durablement des acquis aussi solides. Le mouvement pour l'assainissement de la santé publique avait été bien lancé. l'administration de Hingston est la part de budget consacré à ce secteur. Alors qu'au 
cours de la fin des années 1860 (1868-1870), malgré les efforts de Workman, le budget du Bureau de santé était en moyenne d'environ 6000 \$ (sur un budget total de 407563 ), il passe à $25000 \$$ en 1876 pour retomber à $17000 \$$ en 1877 et $11000 \$$ en 1875 sous l'administration Beaudry ${ }^{19}$. A noter cependant que les années où il $\mathrm{y}$ a les dépenses les plus élevées ne sont pas nécessairement celles où la santé de la population est la meilleure; c'est même le contraire, puisque les pics correspondent aux années d'épidémies de variole très graves (1875-76 et 1885-86). Il est aussi compréhensible que les dépenses baissent quand on a pu enrayer l'épidémie comme c'est le cas pour la variole après 1877 sous les deux mandats de Beaudry.

Durant toute cette époque, le bureau de santé doit être nettement distingué du département de santé, un des départements administratifs de la municipalité. Le Bureau de santé ou Commission d'hygiène est formé d'un président, qui est le maire et de certains échevins. A partir de 1875, A.B. Larocque, premier officier de santé à plein-temps de la ville, prépare un rapport annuel sur l'état sanitaire de la ville qu'il soumet au président et aux membres du bureau de santé. Régulièrement, dans sa lettre d'introduction, il réclame la constitution d'une organisation complète de son département sur le modèle de ce qui se fait à l'époque dans les principales villes d'Europe (principalement en Grande-Bretagne) et des États-Unis ${ }^{20}$.

En plus de l'officier de santé, le département est composé en 1880 de quatre inspecteurs qui font chaque année un recensement sanitaire de la population centré sur la salubrité des logements et le recensement des personnes vaccinées contre la variole. Dès cette année-là l'officier réclame le service d'un ingénieur sanitaire.

31 La ville continue à promouvoir la vaccination et à embaucher des médecins vaccinateurs vacataires, à raison d'un par district. A partir de 1880 le vaccin est produit sur place par un médecin de la ville qui entretient des génisses pour cela ${ }^{21}$. L'enlèvement des déchets des maisons relève alors d'un entrepreneur privé qui ne fait pas aussi bien le travail que le département des chemins à qui cette tâche était confiée auparavant. En 1876, le contrôle du ramassage des ordures était passé du comité des chemins à celui de la santé mais ceci avait été, semble-t-il, modifié par l'administration Beaudry ${ }^{22}$. Il n'y a pas encore d'inspection des aliments bien que Larocque réclame qu'elle soit confiée à un médecin vétérinaire. Dès 1880 , le discours de l'officier de santé concernant les rapports des maladies contagieuses devient plutôt autoritaire à l'égard des médecins qui « devraient faire rapport des cas de maladies qu'ils pensent avoir à traiter» et surtout des familles pour qui «si elles persistent à refuser de faire rapport au bureau de santé, nous serons forcés de mettre le règlement en vigueur ${ }^{23} »$.

32 A partir des années 1880, Larocque et d'autres membres de la profession médicale vont entreprendre de nombreuses démarches visant à obtenir des législations, fédérales et provinciales, une intervention en matière de santé publique et la création d'un organisme de santé dont la compétence s'étendra au-delà de Montréal ${ }^{24}$. En 1882 le médecin de la cité, aidé d'un comité de médecins et des membres du conseil d'hygiène de la ville, prépare un projet de loi provinciale qui prévoit de créer un conseil d'hygiène provincial, de constituer un conseil d'hygiène local dans chaque cité, ville ou village, de standardiser la statistique vitale à l'échelle de la province ${ }^{25}$. En 1885, dans sa séance du 12 octobre, le conseil municipal, sous la nouvelle direction du maire Beaugrand, élu au printemps de la même année, ratifie l'organisation de ce qu'il appelle le bureau local de santé pour Montréal sous forme d'un conseil d'hygiène ou comité de santé formé d'échevins nommés par le conseil municipal et d'une commission d'hygiène intitulée aussi bureau de santé. 
L'exécutif de ce dernier est composé du docteur Louis Laberge qui succède celle année-là au docteur Larocque comme médecin de la cité, et du docteur Nolin, médecin de l'Hôpital civique. Le personnel à plein-temps comprend quatre médecins vaccinateurs, un inspecteur sanitaire, un secrétaire et un comptable, douze agents de police sanitaire, un agent pour la désinfection, deux inspecteurs de viande, deux inspecteurs aux abattoirs, cinq commis dont un messager, et un garçon de bureau. Le personnel adjoint temporaire est aussi nombreux comprenant quatre médecins vérificateurs, douze médecins vaccinateurs, trois médecins aux hôpitaux des varioleux, dix personnes pour la désinfection, treize pour les affiches, quatorze commis dont quatre pour le bureau d'isolement. L'importance de ce personnel s'explique en partie par la «formidable épidémie variolique qui vient de visiter la ville » et qui vient de faire plusieurs milliers de victimes mais également comme un résultat des efforts cumulés depuis Workman et Hingston pour mettre sur pied un service de santé efficace ${ }^{26}$.

En 1892, la structure du bureau est renforcée par la création d'un poste d'ingénieur sanitaire. Deux ans plus tard, l'entrée de Joseph Edouard Laberge comme bactériologiste marque les premiers efforts d'introduction des nouvelles découvertes scientifiques dans la lutte contre les maladies contagieuses. En 1905, le Service des maladies contagieuses, dirigé par ce dernier, est créé. L'inspection des écoles, qui constituera sa réalisation principale, commence l'année suivante.

\section{Réforme des années 1910 et naissance d'une bureaucratie du service de santé}

34 Au début des années 1910, alors que le docteur Louis Laberge est toujours médecin de la cité, le bureau d'hygiène et de statistique est composé de quatre services. Le Service des statistiques vitales relevant directement du médecin de la cité comprend un médecin statisticien, un secrétaire et deux autres personnes chargées de l'enregistrement des naissances et des décès. Le Service de l'inspection sanitaire est dirigé par un ingénieur sanitaire qui a sous ses ordres dix-sept agents inspecteurs dont quinze sont affectés à la surveillance des quartiers appelés districts de la ville, les autres étant spécialisés dans l'inspection de la plomberie. Le Service de l'inspection des denrées alimentaires, avec vingt-cinq inspecteurs, contrôle le lait chez les producteurs et les vendeurs cherchant à détecter le mouillage et à vérifier la qualité bactériologique. La viande est elle aussi surveillée sur les lieux de vente mais aussi d'abattage. Le Service des maladies contagieuses a pris de l'ampleur avec l'inspection médicale des écoles, qui emploie dixneuf médecins inspecteurs.

En 1915, malgré la guerre qui mobilise certains agents, le nombre des fonctionnaires et employés du bureau municipal d'hygiène et de statistique s'élève à cent soixante et onze personnes. L'année précédente le Service de l'inspection des denrées alimentaires s'est scindé en deux. Devant l'importance de la mortalité infantile par diarrhée (41.9\% en moyenne du total des décès chez les enfants en dessous de deux ans entre 1905 et 1915) une offensive est menée par le bureau d'hygiène pour améliorer l'approvisionnement en lait en autonomisant un Service d'inspection du lait et des fermes. Un corps d'inpecteurs ruraux est créé pour mieux contrôler la production du lait et de la crème. En 1914 le Service de l'inspection sanitaire se réorganise également pour améliorer l'efficacité des inspecteurs dont le nombre a plus que doublé depuis 1910. La plus grande partie d'entre 
eux est encore responsable d'un district mais on assiste à une spécialisation de certains agents chargés d'inspecter plus particulièrement les logements et les établissements industriels. L'extension du collecteur d'égouts permet de supprimer beaucoup de fosses d'aisance bien que celles-ci demeurent la seule ressource dans des quartiers non atteints par le réseau comme Rosemont, Emard et Bordeaux ${ }^{27}$.

En 1914 la ville de Montréal dépense $1.74 \%$ de son budget pour son département d'hygiène, 14.99 \% pour l'ensemble de l'hygiène et de la salubrité publique (entretien des hôpitaux, nettoyage des rues, fournitures de l'eau et bain public), $15.4 \%$ si l'on ajoute l'entretien et la réparation des égouts. La ville dépense par ailleurs $2.54 \%$ de son budget aux fins de charité, $15.65 \%$ pour l'éducation, $18.20 \%$ pour la police, les incendies et l'éclairage, $5.67 \%$ pour l'administration et surtout $32.5 \%$ pour le service de la dette ${ }^{28}$.

En 1914 apparaissent deux nouveaux services autonomes, celui des laboratoires et du contrôle médical, le premier officialisant l'importance des "analystes de la ville », le second ayant pour mission de contrôler la santé des fonctionnaires de la ville.

En 1916, un Service municipal anti-tuberculeux à la charge d'un médecin (le docteur H. Gervais) et de deux inspecteurs est inauguré à l'intérieur du Service des maladies contagieuses avec deux consultations de dispensaires qui viennent renforcer celles des instituts Royal Edward et Bruchési. En 1917, avec l'arrivée du docteur Eugène Gagnon, un embryon de Service d'hygiène infantile apparaît. En 1918, le bureau d'hygiène croît brutalement avec l'absorption du département de l'Assistance municipale, ainsi que différents services appartenant à d'autres départements municipaux comme les parcs et jardins. Ceci va motiver sa réorganisation totale sous forme de divisions. Le nouveau Service de santé qui prend définitivement ce nom jusqu'à la fusion en 1972 du Service de santé et du Service de l'assistance municipale, est alors composé de neuf divisions. Elles demeureront sensiblement les mêmes durant le demi siècle qui va suivre, si ce n'est le transfert, en 1922 d'abord, de la division des récréations publiques, puis en 1940, de l'assistance municipale rattachée au nouveau Service du bien-être social créé à cette époque. La nouvelle division de l'hygiène de l'enfance dirigée par le docteur Eugène Gagnon prend de l'importance en absorbant l'inspection médicale des écoles, son travail portant « dorénavant sur un cycle complet de surveillance sur l'enfant depuis avant (sic) sa naissance jusqu'à sa sortie de l'école ${ }^{29}$ ». Le personnel du service de santé passe de 130 fonctionnaires et employés au début de 1918 à 210 au 31 décembre la même année, doublant presque ses effectifs.

\section{La crise provoquée en 1927 par l'épidémie de fièvre typhoïde et l'enquête de 1928}

39 L'épidémie de fièvre typhoïde qui sévit à Montréal durant la fin de l'été et le début de l'automne 1927 n'est pas la première à survenir dans la citée ${ }^{30}$. Cependant, de par son ampleur et sa gravité elle va déclencher la nomination d'un comité d'enquête chargé de faire un rapport sur l'hygiène dans la ville de Montréal, à l'initiative de Sir Arthur Currie, président de la Ligue de Montréal contre la tuberculose et pour la santé publique et composé d'hommes d'affaires et de professionnels comme les docteurs Martin et Harwood mais aussi de personnalités du monde universitaire comme Edouard Montpetit. Environ 5,000 cas et 533 décès sont à déplorer, un mauvais contrôle de la pasteurisation du lait étant en cause ${ }^{31}$. 
40 En octobre 1928, la Compagnie d'assurance-vie métropolitaine publie son rapport d'enquête sur les activités en hygiène publique à Montréal. Il s'agit d'une véritable évaluation réalisée en réalité non par le comité d'enquête lui-même mais par un comité technique composé de spécialistes montréalais en hygiène publique : les docteurs J. A. Baudoin, A. Grant Fleming et R. St. MacDonald, qui sont supervisés eux-mêmes par des consultants de l'American Public Health Association dont le plus éminent est C.E.A. Winslow. Les experts utilisent une formule normative préparée en 1925 par le " Committee on Administrative Practice of the American Public Health Association » pour évaluer les services de santé municipaux. Se basant sur les activités à la fois des services publics et des associations privées, la quantification au moyen de cette grille de l'APHA fait ressortir les points forts et les points faibles du Service de santé de Montréal.

41 Parmi les points forts, le rapport souligne principalement tout ce qui se rapporte à la salubrité (eau et égouts), mais aussi à l'hygiène maternelle et prénatale ainsi qu'à l'hygiène préscolaire. Concernant la salubrité, les experts s'interrogent sur le bien-fondé du rattachement au Service de santé plutôt qu'au département de la construction de la municipalité de l'approbation des plans de construction et de l'inspection de la plomberie dans les constructions nouvelles. Indiquant que $100 \%$ des logements sont pourvus d'une eau de la qualité requise et que $99.5 \%$ d'entre eux sont reliés à la canalisation des égouts, ils considèrent que les activités du service en faveur de la salubrité (inspection sanitaire, eau et égouts) sont très satisfaisantes. Ils reconnaissent également comme excellent le travail du comité d'urbanisme de la Ligue du progrès civique en faveur d'une loi d'urbanisme à l'échelon provincial, encadrant un règlement municipal concernant l'habitation. Ils recommandent une meilleure collaboration avec le Service provincial d'hygiène pour surveiller les puits de l'île de Montréal souvent utilisés par les citoyens pendant les mois d'été.

42 Si la cote donnée par les experts à l'hygiène maternelle est élevée, ce n'est pas seulement à cause du service municipal de santé. De nombreuses autres organisations participent à cette action comme l'Assistance maternelle, le Royal Victoria Montreal Maternity Hospital, les centres de langue française et de langue anglaise de la Ligue de Montréal contre la tuberculose et pour la santé publique, la Child Welfare Association, le Victorian Order of Nurses et la Compagnie d'assurance-vie métropolitaine par leurs infirmières visiteuses à domicile. $35 \%$ des grossesses sont alors suivies à domicile avec 3 visites en moyenne par cas. La mortalité maternelle, qui est de 3,8 pour 1000 , est l'une des plus basses du Canada, bien que 16 \% seulement des naissances surviennent dans les hôpitaux.

43 La situation est moins satisfaisante dans le domaine de l'hygiène de l'enfance. La mortalité infantile demeure élevée - 127 pour 1000 en 1927 - surtout si on la compare avec celle des grandes villes nord-américaines. Sur environ 21000 naissances vivantes survenues en 1927, 8700 auraient été visitées à domicile dès le premier mois par une infirmière et 15600 après le $1^{\mathrm{er}}$ mois avec une moyenne de deux visites par enfant. Par ailleurs 8739 nourrissons, soit $41 \%$ des naissances à Montréal, auraient été suivis en consultation au moins une fois, mais le comité signale que lors de ces consultations dont celles du Service de santé municipal - « on n'a pas adopté l'habitude de déshabiller les enfants pour les mettre sur la balance pour les soumettre à l'examen du médecin ${ }^{32}$ ». Se basant sur les inégalités ethniques concernant le risque de mortalité infantile, les experts recommandent qu'« après une étude des taux de la mortalité infantile par quartier, il soit ouvert de nouvelles consultations dans les quartiers qui présentent des taux élevés, et qui ne sont pas actuellement desservis ${ }^{33} »$. 

le comité est sévère à l'égard de l'hygiène scolaire qui n'atteint pas $50 \%$ de la norme (selon leur barème une ville avec un service de santé compétent dépasse 90 \%). Malgré les services de douze médecins à plein temps et de trois médecins à temps partiel ainsi que de quarante infirmières scolaires, de nombreuses insuffisances sont signalées. Le manque de coopération entre les autorités municipales et scolaires est relevé de même que l'insuffisance d'infirmières : une pour 3300 élèves correspondant approximativement à sept écoles alors qu'il en faudrait une pour 2500 enfants. Sur la base du constat que $47.9 \%$ seulement des élèves sont trouvés sans carie dentaire, l'indigence du service dentaire est également dénoncée: il n'y a qu'un seul dentiste scolaire alors qu'il en faudrait vingt. D'autres carences dans l'organisation et les moyens de ce service sont soulignées comme le manque de balances: (trente pour 255 écoles), l'absence de local réservé à l'hygiène scolaire, la non-implication des enseignants et des parents dans la tenue des cartes de santé et l'examen de dépistage, et surtout, l'insuffisance de l'enseignement de l'hygiène. Le comité recommande en particulier que «l'enseignement de l'hygiène relève des autorités scolaires tout autant que l'enseignement de toute autre nature ${ }^{34}$ ».$$
\text { le retard de la notification des naissances et les contradictions concernant les chiffres de }
$$
naissance et de décès selon les données municipales, provinciales ou fédérales, l'organisation du laboratoire municipal $d$ 'hygiène est jugée très insuffisante. Les auteurs du rapport recommandent la création d'une division des rapports et des statistiques dans le Service de santé, ce qui aura lieu l'année suivante, le docteur Eugène Gagnon étant nommé surintendant de la division de la démographie en octobre 1928. La surveillance bactériologique et chimique de l'eau de consommation est correctement assurée par le laboratoire municipal d'analyses, et elle est complétée par les laboratoires des usines de filtration. Par contre, des défaillances sont notées au niveau des analyses du lait malgré le renfort apporté par le laboratoire provincial. Par ailleurs, le rapport souligne comme point particulièrement négatif, le peu d'initiatives pour entreprendre les enquêtes épidémiologiques. Afin de remédier à cette dernière insuffisance, le comité recommande de décentraliser les points de collecte des échantillons et d'assurer la gratuité de " toutes les analyses reconnues nécessaires en hygiène pour le diagnostic et le contrôle des maladies contagieuses ${ }^{35} »$.

Malgré son ancienneté le système de contrôle de l'approvisionnement des aliments et du lait qui est placé entièrement sous la direction du Service de santé, ne paraît pas aussi efficace qu'on serait en droit de s'attendre, étant donné, en particulier, le grand nombre d'inspecteurs du lait, estimé par le comité "plus élevé qu'il n'est généralement trouvé nécessaire ${ }^{36} »$. Dans ce domaine, les règlements d'hygiène sont récents et le travail d'éducation des employés dans les divers établissements où l'on fait la manipulation des aliments est encore insuffisant.

47 L'enseignement de l'hygiène à la population constitue en effet le talon d'Achille du service, outre le laboratoire municipal d'hygiène dont il a déjà été question. Le comité déplore en particulier l'insuffisance des conférences publiques ou des causeries à des groupes sur des sujets d'hygiène, l'utilisation insuffisante de la radio et la mauvaise coordination des efforts associatifs dans ce domaine de l'éducation populaire. Il recommande la création d'une section d'enseignement de l'hygiène dans la division administrative du service de santé, disposition qui entrera en application dès 1929, avec 
la nomination du docteur Adrien Plouffe comme assistant du directeur et comme responsable de cette section. Des insuffisances sont notées également dans les programmes de contrôle des maladies contagieuses et plus spécifiquement dans ceux pour la tuberculose et des maladies vénériennes. Comparant, par exemple, Montréal à la moyenne de 12 grandes villes nord-américaines, les experts estiment qu'il y a nettement sous-déclaration des cas pour la fièvre typhoïde, la diphtérie, la rougeole, la coqueluche et même la tuberculose pulmonaire. Reproche plus grave, le rapport indique qu'« on ne fait aucun effort systématique pour rechercher la source de l'infection ni les cas qui passent inaperçus ou qui sont cachés, mesures nécessaires au contrôle de la maladie ». La procédure de déclaration est considérée comme trop bureaucratique, beaucoup d'énergie étant gaspillée à recopier les cas sur différentes formules, alors que l'on a déjà enregistré une première déclaration du médecin de famille qui se fait, elle, par téléphone ${ }^{37}$. Estimant que 100000 \$ ont été dépensés indûment en 1927 pour traiter les cas de diphtérie hospitalisés dans les hôpitaux Saint-Paul et Alexandra ${ }^{38}$, le comité déplore que seulement 1010 enfants de un à six ans aient été immunisés cette même année sur les 95000 qui étaient en âge de bénéficier de cette vaccination. La distribution gratuite du sérum antidiphtérique ne se fait qu'aux indigents. De la même manière, alors que l'ophtalmie des nouveau-nés est responsable d'environ $25 \%$ de tous les cas de cécité, il n'existerait aucun programme municipal et provincial pour prévenir cette maladie.

Dans le domaine de la lutte contre les maladies vénériennes, l'implication du Service de santé municipal est très limitée, l'essentiel de l'effort reposant sur le Service provincial d'hygiène qui a dépensé, en 1927, 47388 \$ et reçu du gouvernement fédéral 26 098,74 \$. Il en est de même de la lutte anti-tuberculeuse dont les diverses parties du programme ont été presque complètement déléguées aux organisations privées. Les visites à domicile sont assurées d'abord par les infirmières du Royal Edward Institute et de la Ligue de Montréal contre la tuberculose et pour la santé publique "pourvoyant elle-même à la plus grande partie du service de l'Institut Bruchési ${ }^{39}$ ». Les infirmières du Victorian Order of Nurses et de la Compagnie d'assurance-vie métropolitaine y contribuent également. Le service clinique ambulatoire (diagnostic et traitement) est réalisé essentiellement par les dispensaires des deux instituts Royal Edward et Bruchési ${ }^{40}$. A l'époque l'hospitalisation relève de ce dernier établissement (23 lits) et surtout de l'Hôpital du Sacré-Cœur (350 lits), du Laurentian Sanatorium (250 lits), du Sanatorium Mount Sinaï (45 lits) et de l'Hôpital Grace Dart Home (45 lits). Le rapport recommande non pas la création d'une division de la tuberculose, qui ne sera mise en place que beaucoup plus tard, mais le recrutement d'un médecin spécialiste en tuberculose pour la section d'épidémiologie et de contrôle des maladies contagieuses qui aurait pour fonction de coordonner les différentes ressources et de servir de consultant pour les médecins praticiens. Comptabilisant les dépenses payées pour l'hygiène à Montréal par la ville elle-même mais aussi par le Service provincial d'hygiène et les associations volontaires, l'enquête de 1928 révèle que la dépense par tête-0,69\$-serait inférieure à la moyenne des 12 plus grandes villes des États-Unis ( 0,78 \$ US) et propose de l'augmenter à $0,91 \$$ dans les trois prochaines années.

Dans les années qui vont suivre et parfois, dès 1928, le service va se développer plus rapidement, en s'alignant de plus en plus sur «la conception que l'on avait (alors en Amérique du Nord) de l'organisation de l'hygiène publique et des connaissances scientifiques de la maladie et de la prévention ${ }^{41} »$. La division de l'hygiène de l'enfance en particulier va se développer considérablement, surtout l'hygiène scolaire et l'inspection 
médicale des écoles. Les recommandations sont basées sur des normes qui sont souvent reformulées quant aux mesures de surveillance et de contrôle de la population. L'impact de ce rapport sur la structuration du Service de santé de la ville sera tellement important qu'il inspirera encore les réformes introduites par Adélard Groulx dix ans plus $\operatorname{tard}^{42}$. Cette enquête marque également un virage par rapport au modèle de santé publique qui influence le Service de santé : ce modèle se déplace de l'Europe vers les États-Unis. En somme, la crise provoquée par l'épidémie de fièvre typhoïde de 1927 et les réformes impulsées par le comité d'enquête de 1928 ont conduit à un développement et à une extension des activités du Service de santé municipal au cours des deux décennies suivantes. Toutefois, ce développement des activités du service a été assez inégal selon les secteurs. C'est ce qui va apparaître à la fin des années 1940 dans l'enquête que le gouvernement provincial a fait mener sur le Service de santé de Montréal.

\section{Forces et faiblesses du service de santé municipal montréalais a travers l'enquête provinciale de 1948}

D'après le rapport du docteur J. E. Sylvestre daté du $1^{\text {er }}$ mai 1950, les membres du souscomité technique chargés par le gouvernement provincial d'enquêter sur l'organisation du Service de santé de Montréal estiment que l'administration municipale est « arrivée à un point où elle se demande si elle ne devrait pas obtenir une intense aide du gouvernement supérieur ${ }^{43}$ ». Au 31 décembre 1948 le Service de santé emploie 486 personnes avec un budget de 1543710 \$ pour l'année fiscale 1948-1949 soit 1,50\$ par tête. Pour les auteurs de l'enquête, 257 employés supplémentaires (ce qui représenterait une augmentation de 52,8 \%) seraient nécessaires de même qu'un ajout budgétaire de 975192,71 \$ (63\% en plus), bien qu'un grand nombre d'associations volontaires (le rapport en répertorie 16) dont le budget est estimé à 1062 581,77 \$, interviennent déjà en complémentarité.

Le rapport insiste aussi sur la vocation régionale du service qui intervient par exemple dans le contrôle de la chaîne alimentaire pour le grand Montréal et qui alimente la presse régionale et la radio en campagnes d'éducation sanitaire qui ne profitent pas qu'à la population de la ville. L'importance reconnue du rôle des infirmières, leur grand nombre (186 sur 486, soit $38 \%$ du personnel) la lente montée du féminisme et du professionnalisme en nursing, explique la recommandation des enquêteurs en faveur de la création d'une division du nursing.

Encore presque exclusivement centrée sur la lutte contre les maladies contagieuses, la division du même nom devrait, selon les enquêteurs, changer son nom pour celui de division d'épidémiologie, division qui accorderait plus d'importance à la lutte contre le cancer et les maladies du vieillissement. Malgré les efforts entrepris depuis la deuxième guerre mondiale, la contribution du Service de santé à la lutte anti-vénérienne et même à la lutte anti-tuberculeuse demeure marginale. Les deux divisions sont avant tout des organismes de liaison et de coordination. Dans le domaine de la lutte contre les maladies vénériennes, le contrôle de ces maladies est centralisé depuis la loi de 1941 entre les mains de la division des maladies vénériennes du Ministère provincial de la santé et du bien-être social qui gère directement les dispensaires antivénériens des hôpitaux. Cependant, le Service de santé municipal coopère avec le ministère dans la mise en application des mesures nécessaires à la prévention de ces maladies et à l'éducation du 
public $^{44}$. En ce qui concerne la lutte anti-tuberculeuse, le Service de santé s'est d'abord impliqué en subventionnant les deux dispensaires de l'Institut Bruchési et du Royal Edward Institute ainsi que le travail d'éducation réalisé d'abord par la Ligue antituberculeuse et de santé publique entre 1924 et 1928. A partir de 1938, le Service de santé élabore ses propres structures en créant d'abord une section de la tuberculose au sein de la division des maladies contagieuses. La collaboration est très étroite avec le Comité provincial de la tuberculose. La section devient une division en $1948^{45}$.

La division de l'hygiène de l'enfance qui était la plus importante jusqu'à la création des districts sanitaires a vu son personnel être transféré progressivement vers ces derniers. Le premier vrai district sanitaire a été organisé en 1940 autour du quartier de Maisonneuve regroupant trois autres quartiers municipaux. Au fur et à mesure de leur création, les huit à neuf districts qui doivent couvrir toute la ville sont d'abord rattachés à la division de l'hygiène de l'enfance, puis directement au cabinet du directeur avant qu'une division des districts sanitaires ne soit créée en 1947. Tout ce qui concerne l'hygiène maternelle, infantile et pré-scolaire ainsi que l'inspection médicale des écoles est mis en application par le personnel des districts sanitaires mais continue à être planifié par la division de l'enfance. Le réseau des cliniques pré-natales municipales (au nombre de 6) et de consultation municipale infantile (60 en 1948) est toujours complété par dix-huit centres de gouttes de lait paroissiales et huit consultations de la Child Health Association. L'hygiène dentaire qui est l'objet d'une section de la division de l'enfance s'est beaucoup développée depuis l'engagement du premier dentiste inspecteur en 1915. En 1948, dix dentistes à plein-temps et six à temps partiel font un travail de dépistage et d'éducation individuelle ou de groupe dans les écoles, mais aussi dans 11 cliniques dentaires municipales. Les deux divisions de l'inspection sanitaire et de l'inspection des aliments totalisent à elles deux 159 personnes et $424786 \$$, soit près du tiers du budget et du personnel de l'ensemble du service. Ne remettant pas en cause l'appartenance de ces fonctions d'inspection au Service de santé, le comité recommande cependant l'unification de la direction des deux divisions pour éviter les dédoublements.

Comme on le voit, malgré certaines faiblesses persistantes, mais relatives à la comparaison avec certaines autres villes nord-américaines -, le Service de santé de la ville avait continué, dans les années 30 et 40 , à consolider son organisation et à étendre ses activités, avec des résultats indéniables. Ce processus d'extension de l'appareil sanitaire et d'amélioration de la situation de la santé publique à Montréal s'est poursuivi dans les années 1960. Nous allons donc essayer de rendre compte, dans la section suivante et dans la conclusion de ce texte, du paradoxe que constitue le fait que l'on en soit venu à démanteler, dans les années 1970, un service que l'on avait bâti au prix de tant d'efforts et d'initiatives au cours de plusieurs décennies et qui avait largement prouvé son utilité.

\section{Le paradoxe du démantèlement du service de santé}

La décennie 1960 va être marquée par toute une série d'initiatives qui vont dans le sens de la médecine globale et d'une concentration du personnel dans les districts les plus défavorisés. Jusqu'à cette période le Service de santé offrait une médecine quasi exclusivement préventive. C'est principalement dans les consultations des districts de Saint-Jacques Est et Ouest qui, comme on l'a vu, sont parmi les districts les plus défavorisés de la ville - que la médecine globale va être mise en opération. Cette mise en place est freinée par des résistances internes liées au manque de formation du personnel 
à ces nouvelles tâches et surtout par les divergences entre les responsables du Service de santé qui souhaitent s'adresser d'abord aux indigents au travers de leur programme spécial en milieu défavorisé et les comités de citoyens qui réclament une véritable assurance-maladie pour tous ${ }^{46}$.

En 1968, un projet pilote est implanté dans le district sanitaire de Saint-Jacques autour «d'un nouveau concept de la médecine préventive dans la communauté urbaine ${ }^{47}$ ». Il s'agit en réalité d'offrir aux mères d'une zone défavorisée des services de dépistage gynécologique et de planification familiale avec fourniture gratuite de produits anticonceptionnels et des médicaments de base. L'expérience sera élargie sous forme d'un programme spécial de soins médicaux dans des écoles en milieu défavorisé appelé " projet renouveau ». Intervention de type « Médecine globale » avant que la Commission d'enquête sur la santé et le bien-être social n'en popularise le nom, il s'agit de dispenser dans ces milieux des «soins médicaux complets préventifs et curatifs tels que examen médical, physique et mental, dépistage, diagnostic temporaire, soins préventifs, prescriptions, traitements, hospitalisation (si nécessaire), relance, visite à domicile, réadaptation et éducation sanitaire ${ }^{48} »$. Autorisés par le Collège des médecins de la province de Québec à agir comme médecins traitants en milieu défavorisé à l'emploi de Montréal, les médecins des districts concernés vont bénéficier d'un réseau de soutien hospitalier et d'une subvention du Ministère provincial de la santé pour payer les médicaments. L'approche très professionnelle et normative de ces expériences pilotes contraste avec la démarche empirique et résolument idéologique des «cliniques populaires » comme la clinique des citoyens de Saint-Jacques ${ }^{49}$.

(a) se prentait comme une application des recommandations suggérées par la $\mathrm{CESBES}^{50}$. Elle avait été appelée de tous ses vœux par l'Association des médecins du Service de santé de Montréal. Celle-ci dans un premier mémoire à la CESBES en avril 1967 souhaitait que l'hôpital devienne un centre communautaire de santé. La séparation traditionnelle entre les services de santé personnels et le service communautaire devrait disparaître pour faire place à un système intégré de santé ${ }^{51}$. Ces vœux seront exaucés par la CESBES qui oubliera totalement les services municipaux de santé ainsi que les unités sanitaires de comté qui seront sauvés in extremis par le Comité sur la prévention de 1972 présidé par le docteur Corbet McDonald. Ce comité donnera naissance aux Départements de santé communautaire qui absorberont les districts comme les structures centrales du Service de santé de Montréal en 1976. Avant ce rattachement, la ville de Montréal avait décidé, en 1972, de fusionner son Service du bien-être social (créé en 1941) et son Service de santé qui étaient devenus alors le Service des affaires sociales. En 1975, le gouvernement provincial décide d'annuler le contrat de service existant entre lui et la ville de Montréal, absorbant ainsi le dernier Service municipal des affaires sociales qui existait encore au Québec ${ }^{52}$.

Dans un premier temps, la ville de Montréal va s'opposer à cette volonté d'intégration aux nouvelles structures mises en place par la réforme des années 1970 : à savoir, les Départements de santé communautaire (DSC) et les Centres locaux de services communautaires $(\mathrm{CLSC})^{53}$. Le comité exécutif de la cité y voit une volonté d'ingérence du gouvernement provincial dans les affaires municipales et de centralisation excessive. C'est l'octroi de subventions provinciales qui va faire pencher la balance en faveur de l'intégration. Ces octrois soulageaient en effet d'autant les finances de la ville qui avait besoin de cet argent pour la réalisation de grands projets dont ceux de l'exposition universelle de 1967. 
59 Après 1976, la ville de Montréal administre encore pour le compte du ministère provincial des affaires sociales ce qui reste du Service municipal des affaires sociales, certaines divisions comme l'inspection des aliments, l'assainissement de l'air et la salubrité générale ayant été transférées à la communauté urbaine de Montréal et d'autres divisions comme celles du nursing, de la médecine préventive et de la santé dentaire, aux six nouveaux DSC qui se partagent le territoire de la ville. Négociée sans enthousiasme par l'équipe du maire Jean Drapeau qui souhaitait conserver la gestion tout en appelant de ses vœux l'intervention financière du gouvernement provincial, l'intégration du Service municipal des Affaires sociales au ministère provincial des affaires sociales sera vigoureusement dénoncée par le Rassemblement des citoyens de Montréal et par le syndicat des fonctionnaires municipaux de Montréal. En particulier, les conseillers de l'opposition au Parti civique du maire, sans nier « les aspects positifs » de la réforme qui « intègre les fonctions de prévention, de traitement et de réadaptation en s'appuyant sur le concept de médecine globale et donne ainsi une nouvelle dimension à la notion de santé publique qu'on appelle maintenant santé communautaire», et tout en reconnaissant aussi le dynamisme des nouveaux DSC, auraient souhaité une entente signée entre ces derniers et la ville, permettant une meilleure décentralisation des services et une véritable participation des citoyens ${ }^{54}$.

\section{Bilan de l'action du service de santé et conclusions}

Au début de la décennie 1970, alors que le Service de santé municipal s'apprête à laisser la place aux structures socio-sanitaires qui se réclament du nouveau paradigme de la médecine globale, l'état de santé des Montréalais et des Montréalaises s'est considérablement amélioré par rapport à la situation qui prévalait au début de la seconde moitié du XIX ${ }^{e}$ siècle. Plusieurs marqueurs épidémiologiques permettent de mesurer ces progrès sans pouvoir cependant les attribuer de manière exclusive aux activités de protection, de prévention et de promotion de la santé mises en œuvre dans la cité de Montréal tout au long de cette période. Le taux de mortalité infantile constitue l'un des meilleurs marqueurs des progrès réalisés. Comme l'indique la figure 1 , ces progrès paraissent surtout s'amorcer à partir de la première guerre mondiale. Ils s'accélèrent après cette guerre pour finalement diminuer très régulièrement, sans cassure pouvant évoquer une intervention plus spécifique du Service de santé à un moment déterminé. Il s'agit plutôt des effets d'une intervention régulière. Les gains concernant les décès par diarrhée évitables se présentent à peu près de la même manière évoquant des interventions lentes à fournir des résultats mais constantes.

Les compilations par mois et selon les groupes ethniques dominants font ressortir les mêmes constatations que celles qui avaient été observées au milieu du XIX ${ }^{\mathrm{e}} \mathrm{s}$. Il existe un net excès de mortalité pendant l'été. Les disparités sociales dans la distribution des taux constatées au XIX ${ }^{\mathrm{e}} \mathrm{s}$. sont toujours là au XX $\mathrm{e}$. En 1929, la mortalité infantile plafonne à $148,7 \%$ chez les Canadiens français alors qu'elle est de 71 chez les Canadiens britanniques et de 25,91 dans la communauté juive. En 1971, la mosaïque ethnique s'est modifiée mais des déséquilibres subsistent, le taux de mortalité infantile étant, à cette date, le plus élevé dans la communauté d'origine britannique $(25,5 \%$ ) suivie de la communauté d'origine française $(19,2$ pour 1000$)$ puis italienne $(17,2 \%)$ et juive $(11,2 \%)$. Il avait fallu attendre la naissance des districts sanitaires, à partir de 1938, pour voir apparaître des tableaux et des cartes mettant en relief les disparités des taux de mortalité 
infantile selon les quartiers. En 1971, la mortalité infantile est encore de $33 \%$ dans le district de Saint-Jacques et 27,6\%o dans celui du Sud-Ouest (Saint-Henri et Saint-Paul, Ville Emard) alors qu'elle est déjà seulement de $11,7 \%$ à Notre-Dame de Grâce. Le discours des responsables semble s'intéresser plus à « l'excédent de nourrissons » attendu de la lutte contre la mortalité qu'aux déterminants de cette mortalité bien que tout le discours éducatif plaide en faveur d'une compréhension des facteurs culturels - et pas seulement économiques - qui sont probablement à l'origine des disparités interethniques et entre quartiers. Les stratégies d'intervention en faveur des quartiers défavorisés, qu'on n'avait pas encore appelés groupes ou populations à risque, apparaîtront seulement au milieu de la décennie 1960, peu avant la fusion du Service de santé avec celui du bien-être social.

Pour ce qui est de la lutte contre la mortalité maternelle, le dernier directeur du Service de santé exprime ouvertement sa satisfaction : «En 1971 le taux de mortalité maternelle est le plus faible jamais atteint dans les annales du Service de santé de Montréal. » Le tout est de 0,5 pour 10000 naissances, c'est-à-dire un décès pour 18426 accouchements de mères domiciliées à Montréal. Pour le directeur du service: "Ce résultat nous laisse présumer que non seulement les femmes enceintes fréquentent régulièrement les classes pré-natales et les cliniques d'hygiène maternelle mais que les soins obstétricaux dispensés dans les hôpitaux et les maternités sont efficaces et de bonne qualité même en milieux défavorisés ${ }^{55}$. Là encore, si à partir de la fin des années 1960, un mouvement s'amorce pour mettre en place des cliniques spéciales d'hygiène maternelle auprès de femmes défavorisées, spécialement les résidentes des districts centre-ville et centre-sud, le Service de santé, pendant longtemps, s'est intéressé à la lutte contre la mortalité maternelle sans considération de groupes à risque.

L'évolution de la mortalité par tuberculose constitue également un marqueur satisfaisant des progrès dans la lutte contre cette maladie sans que l'on puisse cependant les attribuer uniquement aux activités spécifiques de la section puis de la division de la tuberculose. Malgré les réserves à formuler concernant l'exhaustivité et la fiabilité des diagnostics de décès par tuberculose, surtout pulmonaire, les données secondaires fournies par les rapports et les bulletins permettent de dresser un portrait saisissant de l'évolution de la mortalité par tuberculose pour les deux sexes et tous âges réunis (figure 2). Les deux pics de décroissance observés autour de 1920 puis de 1950 seront confrontés ultérieurement avec les données plus précises concernant la mise en œuvre de certains moyens de lutte comme le sanatorium ou la collapsothérapie, la chimiothérapie anti-tuberculeuse et la vaccination par le BCG.

L'évolution de la mortalité par diphtérie est encore plus saisissante. Partant de 38,8 décès pour 10000 habitants en 1914, le taux de mortalité devient nul en 1963. La pente de la courbe paraît présenter deux inflexions principales, la première correspondant à la sérothérapie dès le début $\mathrm{du} \mathrm{xx}^{\mathrm{e}}$ siècle, la seconde à la vaccination massive à partir des années 1930 (figure 3), mesures sanitaires mises en œuvre par le Service de santé. Ces hypothèses ont besoin cependant d'être consolidées par une analyse plus précise de la couverture vaccinale et de l'extension de l'utilisation de la sérothérapie, ce qui est en cours de réalisation.

65 Avec les années, la répartition de la mortalité par causes va se modifier profondément. Bien que la description de l'évolution de la mortalité par cancer et maladies de cœur exige une analyse encore à faire des modalités de classification propre à chaque grande période, les grandes tendances ressortent nettement du tableau II. Le cancer qui occupe le 
rang 8 parmi les 10 principales causes de décès en 1914 avec un taux de 68,8 pour 100000 habitants passe au deuxième rang en 1963 avec un taux de 168,9 pour 100000 habitants. Les rapports annuels du Service de santé permettent de vérifier cette lente mais inexorable montée du cancer depuis la fin du XIX ${ }^{\mathrm{e}}$ siècle mais les taux reproduits auraient besoin d'être vérifiés après standardisation pour l'âge. Les données concernant les maladies du cœur sont encore plus difficiles à interpréter, les cardiopathies ischémiques ayant probablement pris la place très progressivement des valvulopathies. Le taux de mortalité par maladies du cœur qui occupait la cinquième place en 1914 (107 pour 100000 habitants) a déjà pris la première en 1963 (319 pour 100000 habitants).

Ces nouveaux problèmes ne vont s'imposer qu'avec retard, car les responsables du Service de santé de Montréal étaient encore - et on le comprend-très activement engagés dans la lutte contre les maladies contagieuses comme la vaccination contre la poliomyélite. Il faudra attendre la création des DSC pour voir apparaître un nouveau discours qui s'attaque aux habitudes de vie et aux facteurs de risque des maladies cardiovasculaires par athéromatose et des cancers. Le Service de santé de Montréal aurait-il pris avec trop de retard le virage épidémiologique que la nouvelle santé communautaire imposera durant la décennie 1970 ? L'analyse de la période de fusion du Service de santé avec celui du bien-être social et le transfert vers les DSC au cours de cette décennie nous révèle que la "démunicipalisation » du Service de santé ne s'est pas faite sans résistance de la part du personnel comme des autorités municipales. Bien que ces derniers aient souhaité l'intervention de L'État provincial pour des raisons financières, elles souhaitaient, comme les membres de l'opposition à l'équipe du parti civique au pouvoir, le maintien d'une gestion décentralisée. Pour le nouveau Ministère des affaires sociales, il était impensable de financer les nouvelles structures de santé publique sans les contrôler directement. En sectorisant le territoire de la ville avec les nouveaux DSC, un meilleur quadrillage sanitaire de la région devenait possible. La nouvelle idéologie de la médecine globale fit le reste en créant un consensus apparent des professionnels de l'ancien Service de santé, des technocrates de la santé communautaire et des groupes de pression populaire autour des structures nouvelles : les CLSC et les DSC.

TABLEAU 1 
Évolution comparée de la population de Montréal (ville, île et région) d'après les recensements nationaux

\begin{tabular}{|c|c|c|c|}
\hline & Vill|le: & $\|$ & Fingrion \\
\hline $18185: 1$ & 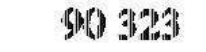 & ㄴ.18 0.15 & \\
\hline 1841 & 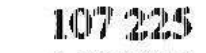 & 14.0144 & \\
\hline$\| 601$ & 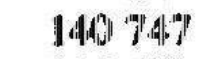 & 192157. & \\
\hline 13911 & 1640 & 279525 & \\
\hline 19011 & 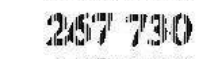 & 3150.938 & \\
\hline$\|9\| \|$ & 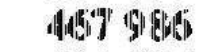 & 434701 & \\
\hline \|19:1 & (4) 180 & 7243015 & \\
\hline$\|93\|$ & Q118 & 1003 istow & $\| 023 \quad 150$ \\
\hline $194: 1$ & $90(3)$ on:? & \|\| $15,8000$ & $\| \quad 139921$ \\
\hline$\|95\|$ & 11 0ad 520 & 1 $3200+372$ & 13954 \\
\hline$\| 946: 1$ & 1. 19.91062 & \& 74.796969 & 21099 \\
\hline 1971 & I) $2 \| 4132$ & $1999 \quad 1.43$ & 27432018 \\
\hline
\end{tabular}

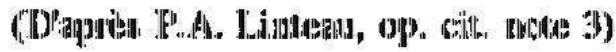

Tableau 2

Évolution comparée des dix principales causes de décès en 1914 et en 1963 à Montréal

\begin{tabular}{|c|c|c|c|c|c|}
\hline \multirow[t]{2}{*}{ Raug } & \multirow[t]{2}{*}{$\begin{array}{l}\text { Couseas } \\
\text { de discis }\end{array}$} & \multicolumn{2}{|c|}{ 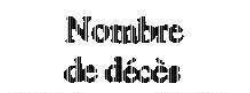 } & \multicolumn{2}{|c|}{ 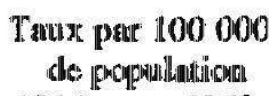 } \\
\hline & & 19144 & 1963 & $1914^{4}$ & 1.9463 \\
\hline 1 & Diesoch he et endicritive & 2.293 & 12 & 446,9 & 0,5 \\
\hline 2 & Tubercullose & 1. 160 & 104 & 226,1 & 8,5 \\
\hline 3 & Pneumomie & 1. 0116 & 138 & 107,0 & 11,3 \\
\hline 4 & Misladives de la & & & & \\
\hline 5 & 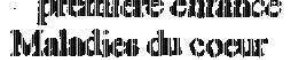 & $\begin{array}{l}952 \\
549\end{array}$ & $\begin{array}{r}375 \\
3901\end{array}$ & $\begin{array}{l}185,5 \\
1070\end{array}$ & $\begin{array}{r}30,7 \\
3100\end{array}$ \\
\hline 6 & Nieglanite & 505 & 229 & 98,4 & $\begin{array}{r}18,7 \\
18,7\end{array}$ \\
\hline 7 & Accidients & 364 & 488 & 71,0 & 39,9 \\
\hline 8 & Cancer: & 353 & 2065 & 68,8 & 168,9 \\
\hline 9 & Léaionas vauculauireat" & & & & \\
\hline 10 & 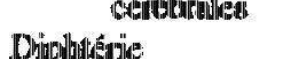 & $\begin{array}{l}3.9494 \\
109\end{array}$ & $\begin{array}{r}782 \\
0\end{array}$ & 67,1 & 63,99 \\
\hline
\end{tabular}

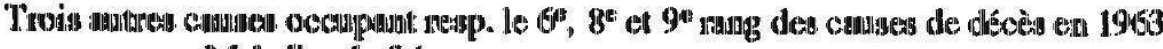
Minlaudien du foie

et de la vësicule $\quad 121 \quad 202 \quad 23,6 \quad 16,5$

$\begin{array}{lllll}\text { Dialbithes } & 46 & 215 & 9,0 & 17,6\end{array}$

Malformutions

$\begin{array}{lllll}\text { conggenitales } & 95 & 170 & 18,5 & 13,9\end{array}$

(D'aprita le Bulleatur d'Hyghidue, L. 3 (1964)) 
figure 1 la mortalité infantile à Montréal (1915-1964)

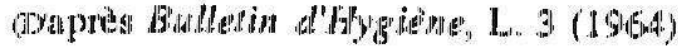

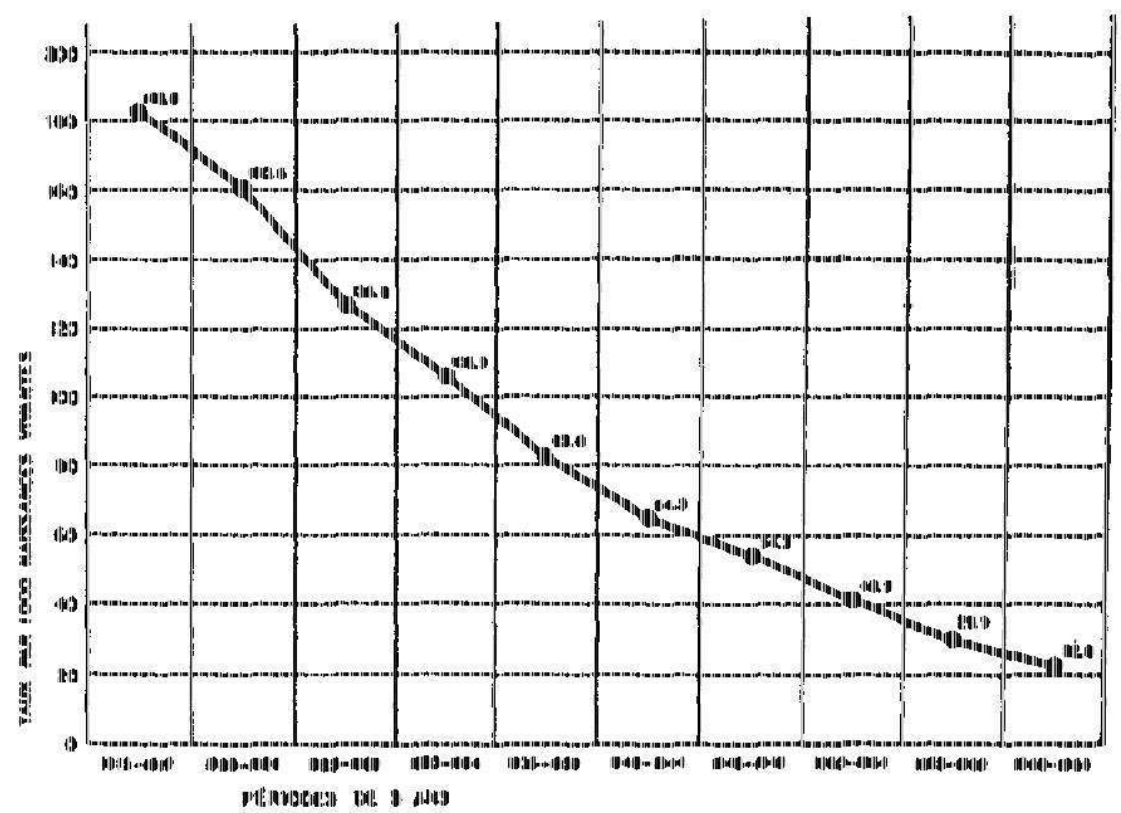

figure 2 la mortalité par tuberculose à Montréal (1915-1964)

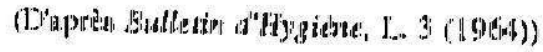

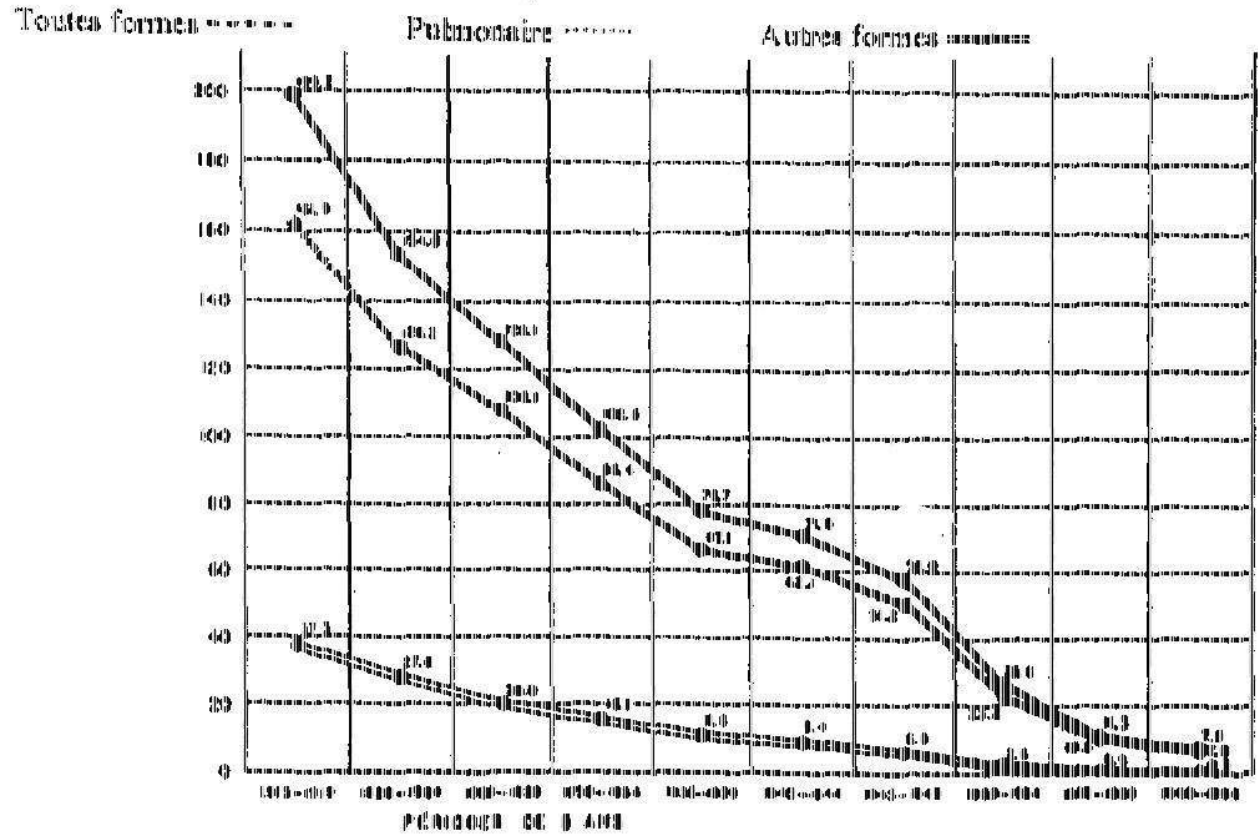


Figure 3 La mortalité par diphtérie à Montréal (1875-1944)

(D'apress Bulletir a'Hygient, XXXX1. 4 (194:5))

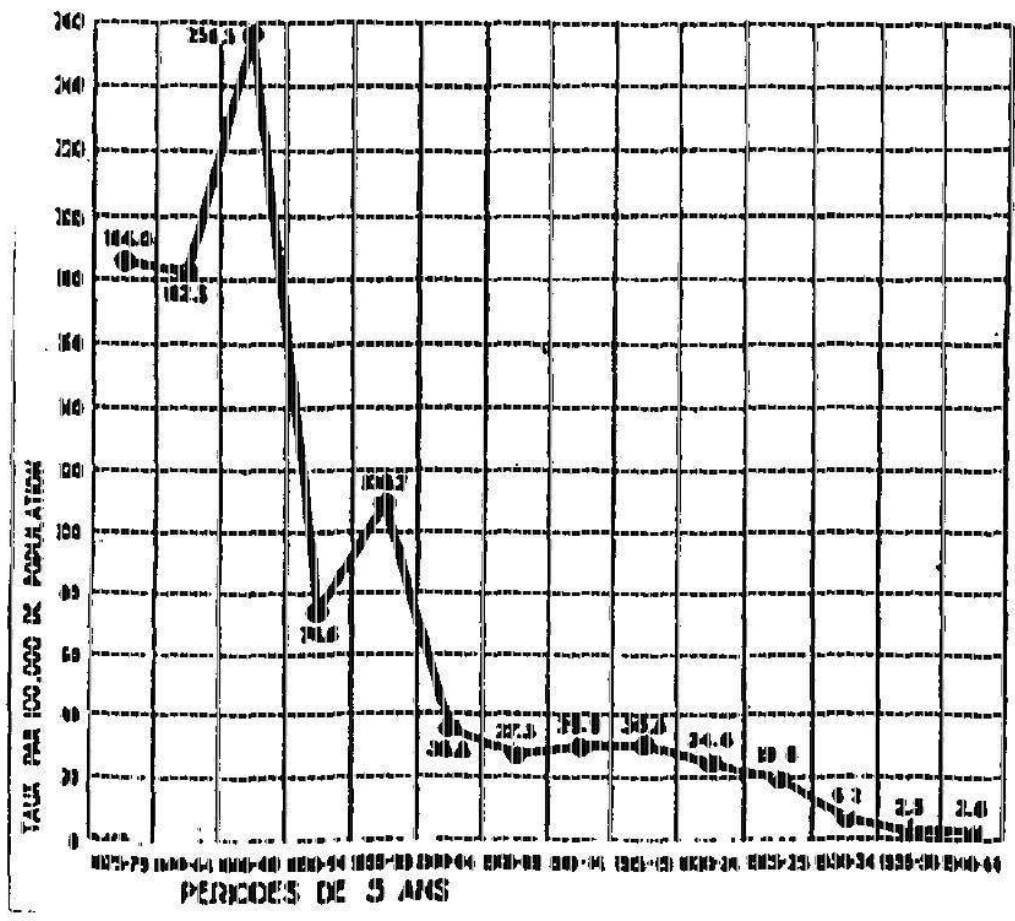

Carte 1 Le Territoire Montréalais à la fin du XIX siècle

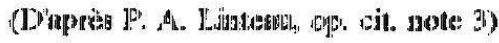

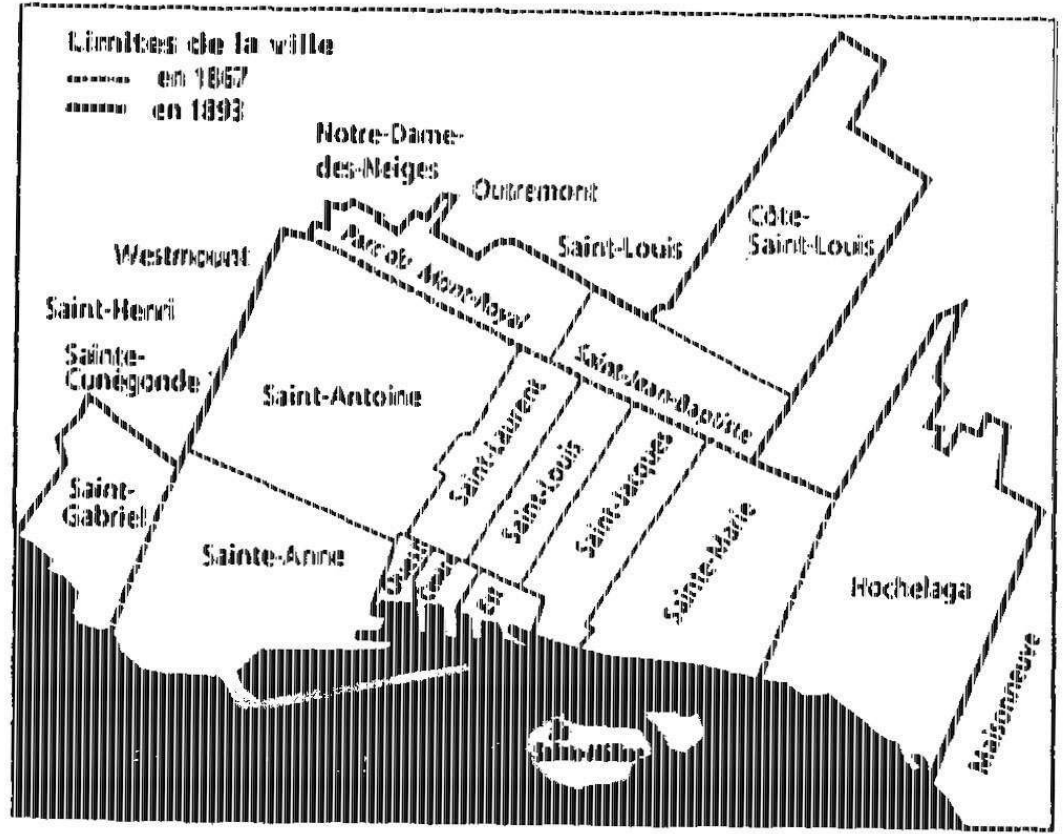


(1) aprè̀s P. A. Linoctent, op. citt, tocolte 3)

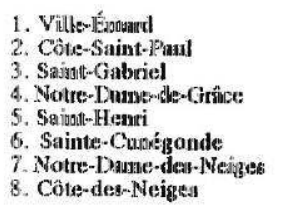

$$
\begin{aligned}
& \text { 9. Parre du Mont Rtoy all } \\
& \text { 10. Satict-Jean-Baptikte } \\
& \text { 11. Saint-Lounis } \\
& \text { 13. Cote-st-Lou } \\
& \text { 14. Vilheriary } \\
& \text { 15. Curtierville } \\
& \text { 16. Bordenaus }
\end{aligned}
$$

17. A.thutetsic

13. Sault-au-Récolet

19. Hochelega

20. Maiscrouneuve

21. Rotsenowat

22. Longus Point

23. Betalarivage

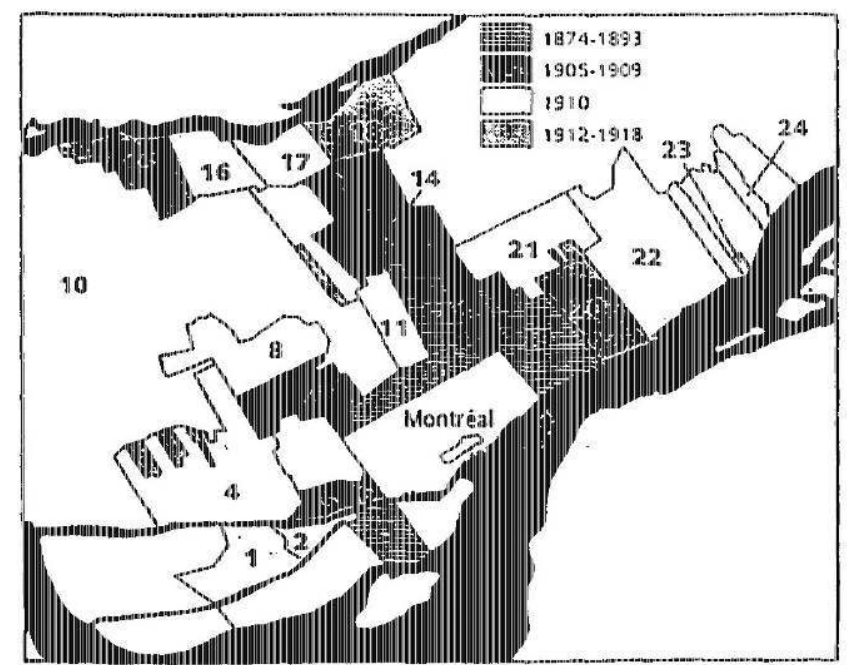

\section{NOTES}

1. John Duffy, A History of Public Health in New York City, 1866-1966, New York: Russel Sage Foundation, 1975.

2. Michael Farley, Othmar Keel et Camille Limoges, «Les commencements de l'administration montréalaise de la santé publique (1965-1885) », HSTC Bulletin, Revue d'histoire des sciences, des techniques et de la médecine au Canada, XX (1982) 24-46 et XXI (1982) 85-109. Une version écourtée et mise à jour a été publiée dans Richard A. Jarrell et James P. Hull (eds), Science, Technology and Medicine in Canada's Past, Thornhill, Ontario : the Scientia Press, 1991.

3. Paul-André Linteau, Histoire de Montréal depuis la Confédération, Montréal : Boréal, 1992.

4. M. Farley et al., op. cit. note 2 .

5. Martin Tétrault, L'état de santé des Montréalais de 1880 à 1914, Mémoire de maîtrise, Département d'histoire, Université de Montréal, 1979.

6. Terry Copp, Classe ouvrière et pauvreté. Les conditions de vie des Montréalais : 1897-1929, Montréal : Boréal Express, 1978.

7. Duffy, op. cit. note 1 .

8. Heather McDougall, Activists and Advocates. Toronto's Health Department : 1883-1983, Toronto et Oxford: Dundurn Press, 1990.

9. Judith Waltzer Leavitt, The Healthiest City : Milwaukee and the Politics of Health Reform, Princeton : Princeton University Press, 1982. 
10. A ce sujet consulter Robert Woods et John Woodward, Urban Diseases and Mortality in Nineteenth Century England, New York: St-Martin's Press, 1984 ; Gretchen A. Condran et Eileen Crimmins Gardner, "Public Health Measures and Mortality in US Cities in the Late Nineteenth Century, Human Ecology, XI (1978) 25-53 ; Robert Woods ", La santé publique en milieu urbain (XIX $-\mathrm{XX}^{\mathrm{e}}$.) : Hygiènes et mesures d'assainissement ", Annales de démographie historique, 1989 ; Thomas Mckeown, The Modern Rise of Population, Londres: Arnold Press, 1976; Simon Szreter, «The Importance of Social Intervention in Britain's Mortality Decline. 1850-1914. A Re-Interpretation of the Role of Public Health », Social History of Medicine, 1 (1988) 1-37 ; Robert Higgs, "Cycle and Trends of Mortality in 18 Large American Cities, 1871-1900 ", Exploration in Economic History, XVI (1979) 381-408.

11. P.A. Linteau, op. cit. note 3.

12. M. Farley et al., op. cit. note 2. Les paragraphes suivants en sont repris.

13. Jean-Claude Robert, "The City of Wealth and Death: Urban Mortality in Montreal 1821-1871 », in J.D. McGinnis et W. Mitchinson (eds), Essays in the History of Canadian Medicine, Toronto, 1988.

14. Patricia Thornton et al., "Dimensions sociales de la mortalité infantile à Montréal au milieu du XIX ${ }^{\mathrm{e}}$ siècle ", Annales de démographie historique, 1988.

15. A.B. Larocque (MD Officier), Rapport sur l'état sanitaire de la Cité de Montréal pour l'année 1879.

16. M. Farley et al., op. cit. note 2, p. 29.

17. M. Farley et al. op cit. note 2 (version mise à jour), p. 279.

18. Concernant l'administration du maire Hingston, voir M. Farley et al., op cit. note 2, p. 32-37 dont ces points ont été repris.

19. M. Farley et al. op. cit. note 2 (version mise à jour), p. 275.

20. A.B. Larocque, Rapport sur l'état sanitaire de la Cité de Montréal pour l'année 1880

21. Ibid.

22. M. Farley et al. op. cit. note 2 (version mise à jour), p. 282.

23. A.B. Larocque, op. cit. note 20.

24. M. Farley et al., op. cit. note 2.

25. Larocque, Rapport de l'état sanitaire de la Cité de Montréal pour l'année 1883, p. 5.

26. Docteur Louis Laberge (médecin de la Cité), Rapport sur l'état sanitaire de la Cité de Montréal pour l'année 1885, p. 7.

27. Docteur S. Boucher, Rapport du Bureau municipal d'hygiène et de statistiques de Montréal pour l'année 1915.

28. Bulletin mensuel du Bureau Municipal d'hygiène et de statistiques, I. 10 (1915), p. 5-7.

29. S. Boucher, Rapport du Service de santé de la Cité de Montréal pour 1918, p. 8.

30. Bulletin d'hygiène, XIII. 7 (1927), p. 1.

31. Bulletin d'hygiène, XIV. 1 (1928), p. 3.

32. Enquête sur les activités en hygiène publique à Montréal publié par la Compagnie d'assurance-vie Métropolitaine, Montréal, 1928, p. 86.

33. Ibid., p. 87.

34. Ibid., p. 99.

35. Ibid., p. 119.

36. Ibid., p. 126.

37. Ibid., p. 52.

38. Ces deux hôpitaux étaient spécialisés dans l'accueil et le traitement des enfants atteints de maladies contagieuses.

39. Enquête, op cit. note 32.

40. Le Royal Edward Institute (fondé en 1909) et l'Institut Bruchési (fondé en 1911) ont été, avec la Ligue antituberculeuse de Montréal (Montreal League for the Prevention of Tuberculosis) (fondée en 1902) les principaux piliers de la lutte contre la tuberculose à Montréal. Cette 
première ligue anti-tuberculeuse de Montréal a d'ailleurs été réincorporée en 1909 sous l'appellation de Royal Edward Institute. (John J. Heagerty Four centuries of Medical History in Canada, II, Toronto : The MacMilan Company of Canada Limited, 1928, p. 231-2.

41. Enquête, op. cit. note 32 .

42. Docteur Adélard Groulx, Rapport du service de santé de la Cité de Montréal PQ (Canada) pour 1938.

43. Enquête sur les Services de santé. T. IV, Services de santé municipaux, 1948.

44. Bulletin d'hygiène, XXXI. 1 (1945).

45. Enquête, op. cit., note 43, p. 77.

46. Robert Boivin, Histoire de la clinique des citoyens de St-Jacques, Montréal : VLB, 1988. Les comités de citoyens sont des regroupements de citoyens qui s'étaient formés spontanément à la base dans la population défavorisée au cours des années 1960. Ils ont pris une importance croissante dans les affaires municipales, en agissant comme groupe de pression revendiquant la mise en œuvre de mesures sociales et progressistes par l'administration municipale. Ils ont également, de leur propre initiative, créé un certain nombre de structures de services médicaux comme les cliniques populaires.

47. Bulletin d'hygiène, LVI. 1 (1970).

48. Ibid., p. 3.

49. Robert Boivin, op. cit. note 46.

50. André Cournoyer, Rapport du Service de santé de Montréal par le directeur pour l'année 1970. CESBES : Commission d'enquête sur la santé et le bien-être social.

51. Premier mémoire présenté à la Commission d'enquête sur la santé et le bien-être social par l'Association des médecins du service de santé de la ville de Montréal, avril 1967. Archives nationales de Québec. Fonds CESBES.

52. Johanne Lapierre, « Nous fêtons cette année le centenaire du Service de santé de la Ville de Montréal », Col blanc, novembre-décembre 1976.

53. Les centres locaux de services communautaires remplaceront, à partir des années 1970, les anciennes unités sanitaires de comité. (Voir Georges Desrosiers, Benoît Gaumer et Othmar Keel, "Vers un système de santé publique au Québec. Histoire des unités sanitaires de comtés: 1926-1975 », Rapport de recherche, Université de Montréal, Département de médecine sociale et préventive et Département d'histoire, 1991. Dans la suite du texte, nous utiliserons les abréviations DSC pour Département de santé communautaire et CLSC pour Centre local de services communautaires.

54. Déclaration des conseillers Ginette Keroak, Paul Cliche et Marius Minier du Rassemblement des citoyens de Montréal, le 9 janvier 1975, archives du DSC de l'Hôpital St-Luc, boîte intégration 1.

55. Bulletin d'hygiène, LVII. 2 (1971). 